\title{
Preparing Nursing Homes for the Fu- ture of Health Information Exchange
}

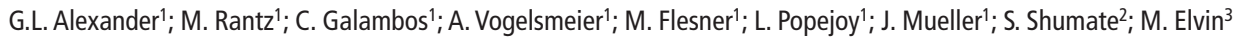
${ }^{1}$ University of Missouri, Columbia, Missouri; ${ }^{2}$ Primaris, Columbia, Missouri; ${ }^{3}$ Professional Services, latric Systems, Inc.

\section{Keywords}

Health information exchange, long-term care, nursing home, health information technology, utilization

\section{Summary}

Objective: Our purpose was to describe how we prepared 16 nursing homes (NHs) for health information exchange (HIE) implementation.

Background: NH HIE connecting internal and external stakeholders are in their infancy. U.S. initiatives are demonstrating HIE use to increase access and securely exchange personal health information to improve patient outcomes.

Method: To achieve our objectives we conducted readiness assessments, performed 32 hours of clinical observation and developed 6 use cases, and conducted semi-structured interviews with 230 participants during 68 site visits to validate use cases and explore HIE.

Results: All $16 \mathrm{NHs}$ had technology available to support resident care. Resident care technologies were integrated much more with internal than external stakeholders. A wide range of technologies were accessible only during administrative office hours. Six non-emergent use cases most commonly communicated by NH staff were: 1) scheduling appointments, 2) laboratory specimen drawing, 3) pharmacy orders and reconciliation, 4) social work discharge planning, 5) admissions and pre-admissions, and 6) pharmacy-medication reconciliation. Emerging themes from semi-structured interviews about use cases included: availability of information technology in clinical settings, accessibility of HIE at the point of care, and policies/procedures for sending/receiving secure personal health information.

Conclusion: We learned that every facility needed additional technological and human resources to build an HIE network. Also, use cases help clinical staff apply theoretical problems of HIE implementation and helps them think through the implications of using HIE to communicate about clinical care.

\section{Correspondence to:}

Gregory L. Alexander

Associate Professor

University of Missouri

Sinclair School of Nursing

Columbia MO 65211

Email: alexanderg@missouri.edu
Appl Clin Inform 2015; 6: 248-266

http://dx.doi.org/10.4338/ACl-2014-12-RA-0113

received: December 11, 2014

accepted: February 27, 2015

published: April 15, 2015

Citation: Alexander GL, Rantz M, Galambos C, Vogelsmeier A, Flesner M, Popejoy L, Mueller J, Shumate S,

Elvin M. Preparing nursing homes for the future of health information exchange. Appl Clin Inf 2015; 6: 248-266

http://dx.doi.org/10.4338/ACI-2014-12-RA-0113 


\section{Background}

Health care providers in over 16,000 nursing homes (NHs) across the United States play an important role in providing care for over 1.3 million residents [1]. Caring for this many residents requires significant amounts of health information exchange (HIE) between a variety of stakeholders including nurses, physicians, pharmacists, and others working both within and outside NHs. HealthIT.gov defines HIE as an information system that, "allows health care professionals and patients to appropriately access and securely share a patient's vital medical information electronically." [2]. Internal and external stakeholders involved in the care of residents use HIE most frequently to monitor resident care tasks, authorize care plans, communicate with each other about patient care, and for financial and administrative activities [3]. Without proper HIEs, health care providers in NHs are at greater risk of breaks in vital communication about resident care, using incomplete clinical data resources, and experiencing limited capacity to make informed care decisions [3]. Additionally, patients are at greater risk for reduced responsiveness to care needs, medical errors, and increased costs from inadequate and fragmented care [4].

Providing access and securely sharing NH resident's personal health information (PHI) through a HIE requires two way electronic communications. PHI refers to demographic information, medical history, test and laboratory results, insurance information and other data that is collected by a health care professional to identify an individual and determine appropriate care [5]. In most NHs, a mix of communication methods including non-electronic and electronic are woven together to support communication between clinicians about PHI [5]. For example, NHs with highly sophisticated information technology systems incorporates interactive electronic status boards to facilitate HIE for internal stakeholders. In these environments, clinicians can post messages electronically to task lists identifying the daily care needs of residents which are documented on by nursing assistants. Clinicians can then track care delivered throughout the day by monitoring electronic charting completed. Licensed nurses and nursing assistants use HIE to share messages about care delivery, such as when patients are turned and repositioned, toileting activities, or notification of a deteriorating skin condition [6]. The same facilities also use non-electronic communication methods to share PHI, for example laminated images and colored dots are posted in close proximity to residents, to easily identify risk status at a glance. Additionally, some of these facilities continue to add resident information to hard copy communication books, such as when residents have had bowel movements. Typically, these books are located in central locations where clinicians congregate to be easily accessible [7].

HIE's used to move information between internal and external stakeholders are still in their infancy. However, many NHs are participating in national initiatives in the United States and abroad to demonstrate the use of HIE to increase access to residents PHI and to transmit PHI securely [3, 4, 8-10]. HIE's facilitate greater communication pathways between internal and external stakeholders to reduce potentially avoidable hospitalizations, improve resident health outcomes, improve transitions of care between hospitals and NHs, and reduce healthcare spending [11]. Lessons learned from these initiatives are being incorporated into new care delivery models to visualize how HIE enhances access to PHI and to improve secure communication systems that will lead to better outcomes [12].

\subsection{Missouri Quality Initiative for Nursing Homes}

The use of HIE is a core component of the Missouri Quality Initiative for Nursing Homes (MOQI), funded by the Centers for Medicare and Medicaid Services (CMS) to reduce avoidable hospital readmission rates in 16 Missouri nursing homes using the MOQI for Nursing Homes Intervention Model [11]. The MOQI model has been described in detail elsewhere, but it involves (a) working with clinicians, health care providers, patients, and families to improve recognition and management of illness, (b) improve quality of care, (c) prepare facilities for implementation and use of HIE, (d) reduce polypharmacy, and (e) improve the use of advance healthcare directives. This paper will specifically focus on preparation phases that facilitated the deployment of a HIE supported by the Missouri Health Connection, one of Missouri's recognized state HIE providers. 


\section{Purpose}

The purpose of this paper is to describe how we prepared 16 NHs for the implementation of HIE. To achieve our mission we used a III phase preparation process in each facility including readiness assessments, development of use cases through field observations, and a formative evaluation using semi-structured interviews to help validate HIE use cases. Formative evaluation is a method used during project development to clarify a theory of change that the project is based on, in this case electronic communication using HIE. The use cases illustrate the potential of HIE use in NH to connect stakeholders involved in resident care. Use cases depict the flow of information between crucial stakeholders involved in resident care decisions who require up-to-date information. Stakeholders include residents/families, clinicians (physicians, nurses, social workers), therapists, dieticians, agencies (pharmacies, medical labs), other NH staff (nursing assistants, transport personnel), in both receiving and transferring facilities.

In this project, use cases help explain complex HIE situations that commonly occur in NHs. Specifically, use cases can be used to support strategic business cases or policy development prior to HIE implementation [13]. Finally, use cases provide a mechanism to visualize current workflow to assure electronic systems incorporating HIE will match anticipated workflows in order to achieve expected outcomes, such as getting information to the right health care provider, in a timely way, in order to improve accuracy of care, and minimize or eradicate medical error.

\section{Sample}

Sixteen NHs were purposefully selected from a Midwest region of the United States in or near St. Louis, Missouri because this geographic location has some of the highest 30-day hospital re-admission rates in the nation, estimated at $20.5 \%$. Specifically, the re-admission rates are high for myocardial infarction, congestive heart failure, and pneumonia diagnoses [14]. Facilities in this project are dually certified Medicare and Medicaid, size ranges from 89 to 321 beds, and the majority [14] are for profit. Facilities are a mix of church affiliated not for profits and corporate for profits. Staffing varies in the facilities, for example, Total Licensed Nursing Hours (RN/DON/LPN) Per Resident Day stretches from 0.72 to 1.48; furthermore, Certified Nurse Aide Hours Per Resident Day ranged from 1.95 to 3.07 .

The following procedures for preparing facilities for HIE implementation were completed between Sept 2012 and June 2014. All procedures were approved by the University of Missouri Institutional Review Board.

\section{Procedures}

\subsection{Phase I Preparation for HIE in Nursing Facilities}

During initial planning stages, prior to implementing HIE, we conducted a brief health information technology (HIT) assessment of each NH facility. The assessment included 22 questions adapted from a validated instrument developed and tested by the author (GLA) to evaluate types of IT used in each $\mathrm{NH}$, the extent of use of IT and level of IT integration with internal and external stakeholders ( Table 1) $[6,15,16]$. Members of our evaluation team also conducted an environmental scan of each facility to determine how accessible computers, printers, and scanners were to NH staff. The environmental scan also included a process to test wireless connectivity throughout the facility, which was noted as complete, partial, or no connectivity.

Following the technology assessment, each facility was asked to identify a system administrator who identified the network of health care providers in each facility who would be involved in conducting HIE. The system administrator also managed access including distribution of user names and passwords with the stakeholders who were assigned electronic mailboxes used for HIE activities. Phase I concluded with a variety of HIE user trainings including onsite face-face sessions, webinars, distribution of reference manuals, and start-up of a 24-hour help desk for user support. 


\subsection{Phase II Preparation for HIE in Nursing Facilities}

During Phase II we conducted observations of 2-3 licensed clinicians and other direct care staff (i.e. transport personnel) in each facility to capture workflow about non-emergent communication strategies used when sharing PHI [17]. Non-emergent situations were selected based on clinician feedback. Clinicians indicated during interviews that information needing immediate attention (emergent situation) would best be communicated directly to a person, over the phone or face-to-face, rather than through an HIE. The rationale was that HIEs are not continuously monitored and emergent information may not be accessed in a timely way. Workflows were mapped into nonemergent use cases depicting communication strategies used by clinicians and direct care staff in these facilities. Additionally, the use cases illustrated the flow of PHI between stakeholders when non-emergent issues were being communicated.

Three team members, including a PhD prepared RN (GLA) with expertise in gerontology and informatics, a project assistant (SS) with a healthcare background, and a consultant (ME) who was an expert in HIE, conducted structured participant observations of clinicians while they were performing clinical work. The team worked with administrators of each $\mathrm{NH}$ to select specific nursing units with residents at high risk of change in condition, where frequent communication between stakeholders was required, typically the unit where Medicare residents were cared for.

For each clinician observed, data were collected that identified events, duration of events, and physical interactions that occurred where resident care was delivered. Additionally, observations focused on specific behaviors associated with communication and documentation strategies used by clinicians during resident care. Individual observations were conducted at approximately 2-4 minute intervals. Total observational periods ranged from 2-3 hours in length and were conducted over two separate visits in each NH. Only observations during the day shifts are included in this study. Observed workflows were used to create use cases that were shown to participants during semistructured interviews conducted in phase III.

\subsection{Phase III Preparation of HIE in Nursing Facilities}

During Phase III we recruited a convenience sample of clinicians to participate in semi-structured interviews from each NH. During the interviews we incorporated a prospective verbal think aloud protocol [17] encouraging these end users to dialogue and provide feedback about the use cases. Clinicians were selected because they play a central role in communication about resident care activities [18]. During these sessions end users strategized how to incorporate HIE into their current workflows. During the verbal think aloud session, the implementation team provided demonstrations of the use of our HIE, called DirectMail, for clinicians as they thought through the various prospects of using HIE to communicate about patient care. DirectMail is a web-based, secure messaging solution similar to email, but encrypted, to route care summaries, clinical notes, lab results and other PHI in a secure, HIPAA-compliant electronic platform [19]. Specifically, participants attending the think aloud sessions were sent messages via DirectMail by the implementation team and asked to respond. After being introduced to DirectMail, clinicians were asked to identify and communicate to team members through the think aloud approach to describe where HIE will enhance the flow of information in a safe and secure way [17]. Use cases developed from clinical workflow observations in Phase II were used to guide discussions during the verbal think aloud approaches with clinicians to determine how HIE could enhance communication in their current processes. Think aloud sessions were recorded and transcribed and were used for developing use cases and thematic analysis. To illustrate discussions about the enhanced flow of information we embedded a "DirectMail" icon into our use cases where participants indicated HIE would enhance flow of information ( $>$ Figures 1-6).

Our motivation for using semi-structured interviews with verbal think aloud approaches was to further understand workflows clinicians use to communicate about non-emergent changes about resident conditions to other clinicians, staff, and families. Additionally, we wanted to understand processes clinicians use to communicate about non-emergent resident conditions to receiving and transferring facilities. We excluded emergent resident conditions because communication about these events are usually conducted immediately and may not be appropriate for HIE (personal email 
(Chuck Crecilius) MD, Medical Director (MOQI)). Each think aloud session consisted of 1-8 voluntary participants. Sessions were conducted with participants from each shift, over a minimum of two days in each of 16 nursing facilities.

\section{Results}

\section{Health Information Technology Assessment}

In these 16 facilities, resident care systems were most integrated with financial management systems used by administrators. Resident care information systems were also integrated with Physical Therapy and Occupational Therapy (PT/OT) disciplines to a greater extent than other resident care disciplines (e.g. pharmacy, dietary, and laboratory). There was very little to no integration of resident care technologies with external entities, which reduces the impact of HIE [20].

All facilities reported having some form of technology available for resident care activities. The majority of facilities (15/16) indicated they used computerized care planning. Just over half (9/16) of facilities indicated they use some form of computerized physician orders transcription process. None of the facilities reported using computerized nursing flow sheets. Only one facility indicated they had computerized historical record keeping, which means that most historical medical records in these facilities are maintained in paper format. Two facilities reported using technology for quality assurance activities. Finally, only one facility reported using technology for clinical reporting, such as for tracking treatments.

There was a wide variety of technology accessibility in resident care areas in these facilities. Half of the facilities (8/16) had computers in resident care areas at the time of our assessment. Five facilities did not have computers, printers, or scanners available in any resident care areas. Six of the 16 facilities had printers available to staff members in nursing areas. Printers that were available, but not in nursing areas, were located in a variety of places including administrative, social services, and education. In these instances some printers could only be accessed during business office hours. Scanners were available in nursing care areas in 3 of the 16 facilities.

None of the 16 facilities had onsite technical IT systems support available to them. Support was provided via external IT contractors who were on call. In several instances, corporate facilities had IT support located in far distant places such as in Illinois or New York. To provide HIE demonstrations our implementation team had to work with IT consultants and contractors to obtain access to wireless networks and to test browser settings at each facility to ensure messages could be sent and received via the HIE. Prior to conducting observations and interviews, problems arose while testing the HIE (Phase I) when configurations included disabled features for the interface that would not allow messages to be sent or received. In the majority of facilities experiencing difficulty these configurations were related to browser settings. Our implementation team included a local Health Information Coordinator that supported these facilities during the testing phases of the HIE. The coordinator worked closely with staff and contractors at each facility for many hours during phase I testing the wireless environment and HIE capabilities in order to demonstrate it to staff. We found that local support is crucial for implementing technology solutions like HIE.

\subsection{Use Cases}

We conducted just over 32 hours of observation of 38 licensed clinicians and direct care staff incorporating different communication strategies used for non-emergent resident care situations in the 16 nursing facilities. From these observations, we determined six HIE activities involving nonemergent resident care were most commonly communicated by staff in these facilities. These six HIE activities or use cases included: 1) scheduling appointments, 2) lab specimen drawing (e.g. antibiotic administration monitoring), 3) pharmacy orders and reconciliation, 4) social work discharge planning communication, 5) admissions and pre-admissions, and 6) pharmacy-medication reconciliation ( $>$ Figures $1-6$ ).

The majority of communication strategies observed in these use cases still involve paper methods such as duplicate carbon copies or fax transmissions. For example, the use case in $>$ Figure 1 sche- 
duling appointments illustrates the sequence of events observed between a NH resident, social worker, clinical staff from the $\mathrm{NH}$, and hospital staff when a doctor's appointment was made for a $\mathrm{NH}$ resident. During this process several paper communication strategies are used including interdepartmental communication forms about the date of the appointment, notification of family members, transportation plans for the appointment, and calendar updates. Paper forms used to prepare for an appointment are shuffled between stakeholders and departments in an effort to coordinate services. Potential HIE problems with paper processes were noted by staff during observations, such as missing information about the reason for the appointment, communication with outside transportation vendors about resident's needs during the transfer. Some of these problems are noted in the use cases.

Every use case includes communication about PHI with external stakeholders. This may be particularly important area for NH HIE since they often lack departments on site such as lab, radiology, or pharmacy. For instance, the use case in $>$ Figure 2 illustrates how lab specimens and results are communicated for residents requiring blood work to monitor drug levels. This sequence of events begins with a nurse confirming a lab specimen draw and result with a receiving medical laboratory. At the time of confirmation the lab verifies with the nurse via cell phone the lab result, when the next lab is due, frequency of lab draws, and types of labs needed. This communication was noted on a post-it note for the next step in the process, which included calling the doctor's office via cell phone to report the lab result, confirm drug dosages and frequency of lab draws. In this use case new orders were obtained and were finally noted in an electronic medical record. Future communications required about this care plan change includes notifying family, doctors signing off on verbal orders, communication to other staff caring for the resident, and the laboratory needs to be made aware.

Use cases provide a method to visualize where HIE can prospectively enhance communication processes. Details in the use cases provide an illustration of the current workflows (e.g. lab results reporting) that can be used to generate discussions and protocols to test process improvements in the future. We used our uses cases to prospectively evaluate where HIE may have the most impact in changing communication about patient care.

\subsection{Semi-Structured Interviews}

Phase III involved the use of semi-structured interviews to validate the findings about the successes and barriers of HIE in health care settings. As part of this process, the six non-emergent use cases were presented and discussed to gain the perspective of the potential clinical users. The MOQI team participated in 68 onsite visits in 16 facilities where we conducted semi-structured interviews ranging in size from 1 to 8 participants per interview. Participants $(\mathrm{N}=230)$ included Licensed and Direct Care Staff [78], Social Workers and Designees [26], Administrators and Directors of Nursing [48], members of the MOQI Team [57], Medical Directors and other health care providers [2], and other types of staff [19]. Semi-structured interviews were transcribed, coded, and analyzed for emerging themes. Three clear themes emerged from the data: 1 . Availability of IT in clinical settings; 2. Accessibility of HIE at the point of care; and 3. Facility management of HIE.

\subsubsection{Availability of IT in clinical settings}

During the interviews, there were repeated statements about the technology needs in facilities which support the findings from our readiness assessment. Not only did participants identify the need for technology but they also offered suggestions for how such technology could be helpful to facility operations. The use of HIE to communicate resident information, to receive new or changing pharmacy orders signed by the physician, and to electronically forward physician orders were some examples that were identified during interviews. Participants emphasized that the use of HIE would be a more efficient means of communication. For instance, HIE would allow multiple stakeholders, like the pharmacy, lab, nursing staff, and even family members, to be notified of changing or new orders simultaneously. Additionally, orders would be more current with physician signatures. One participant expressed "It would help to have enough scanners available with internet capabilities that the forms could be sent via the HIE to all locations at one time and stored electronically." 


\subsubsection{Accessibility of HIE at the point of care}

Participants, at facilities without any IT, articulated access to technology in clinical areas would assist them in carrying out their job responsibilities. Mobile technology was identified as having the potential to be the most useful.

The lack of availability of technology led to workarounds in at least two $\mathrm{NH}$ where some licensed clinicians were using personal mobile phones for texting because, "...that is how the physicians want to be communicated with". In these situations, mobile phones did not have secure messaging to protect PHI sent via this method. In facilities where licensed clinicians were using texting to communicate with physicians, administrators discussed, "concerns about privacy and needing secure solutions to support clinicians at the point of care and to meet physicians' needs". Upon hearing these concerns, administrators who participated in these interviews articulated the need to review policies for communicating with texting. One administrator talked about the need to obtain family members' e-mail addresses upon admission so that better communication can occur with the family members.

\subsubsection{Facility Management of HIE}

The flow of the verbal think aloud approach sparked comments about how to implement and manage HIE. Participants expressed concerns about how to manage the messages including issues related to receiving and sending messages: "how do we know when we receive HIE messages" and "how often do we need to be checking emails?" and again:" what do we do with these messages once we receive them or send them, do we save them in a file somewhere, do they become part of the medical record?"

Concerns were also articulated about how a sender knows that a receiver has read a sent message: "how do we know that someone has read a message that we have sent them through the HIE?" Given the sensitive nature of the information in the HIE, there was some discomfort expressed about not having physical proof that the communication was received and understood by the intended party.

Toward the end of the interviews, participants began to explore the need for facility policies and procedures that would help with the management of communication via the HIE. Suggestions that emerged included the use of an electronic read receipt when HIE messages were sent, which would indicate that a message had been received and opened. Another suggestion that emerged was to save HIE messages into organized file folders set up in the HIE. File folder options in the HIE included setting up folders by patients name or room number then saving messages to the folders.

To handle accessibility issues in resident care facilities that had more technology, participants suggested that the link to the HIE be incorporated on desktops of computers already on nursing units. This arrangement was viewed as a more accessible option which would allow them to have access to the HIE while they were charting, sending, and receiving email, ultimately improving workflow. However participants recognized that such changes enabled a different level of access, which was a concern for administrators. New computer configurations require new policies for setting up and managing computer stations which is another identified barrier to implementing HIE in these settings.

\section{Discussion}

During the preparation phases described we have successfully identified opportunities and challenges to enable HIE implementation in 16 NHs. By incorporating a phased approach including readiness assessments, observations, and semi-structured interviews we have created use cases which are useful for recognizing opportunities and overcoming barriers to HIE implementation.

What we have learned through these approaches is that every one of these facilities needed additional technological and human resources to build an HIE network. These resources cannot be underestimated. Initial costs associated with building an HIE network include the costs of equipment, establishing contracts with vendors who provide the networking capabilities for secure HIE, testing the HIE and establishing appropriate policies and procedures for usage, and ensuring required support staff knowledgeable in IT system configuration are available to meet the changing demands during HIE implementation. Increased and sustained financial and human resources is a 
common finding in other nursing home research exploring technology implementation [21-23]. One limitation is that increased financial and human resources are difficult to realize in nursing home settings, and if they are realized the resources can be difficult to sustain [24].

Through our methodology we also learned that use cases help clinical staff to take a rather theoretical problem of how to use an HIE and helps them think through the implications. Through our observations and conversations with these participants we formed mental models about how HIE could benefit facility staff, residents, families, and stakeholders. We illustrated these mental models in use cases which were matched to clinical workflows that allowed participants to concretely visualize the network. Participants reacted to this approach extremely positively because they began to develop ownership in the design of the HIE and were able to visualize and question the benefits of its use. Published HIE models demonstrate that our use cases created from the mental models of patient care communication are appropriately grounded theoretically $[3,4]$. Use cases with appropriate theoretical approaches provide the basis for real world implementations of HIE in nursing homes, which can guide nursing home leaders as they adopt information technology with HIE capability [25-27].

\section{Limitations}

The number of use cases developed and discussed in this project cannot possibly represent all possible opportunities for HIE, applicable to all facilities. However, with the phased in approach including observations and semi-structured interviews, with feedback by end users, we identified some use cases that have strong face validity established for HIE in these facilities and facilities like them. Other NHs with different types of technologies and workflows would most likely identify other important use cases that could inform possible uses of HIE in their settings. A future goal for project managers and implementation specialist interested in this type of work should be to incorporate our methods to create a bank of use cases that can be used to educate about the benefits of HIE across of wide variety of NHs. Similarly, researchers have indicated that these types of resources would be very beneficial for benchmarking HIE implementation and use [20]. Established use cases will be even more critical as HIE is established between external stakeholders and nursing facilities to avoid pitfalls, such as, delayed care from communication breakdowns due to poor network planning or improper alignment with clinical workflow $[28,29]$.

\section{Conclusion}

We have taken first steps toward understanding the opportunities and challenges of enabling HIE in NHs. Our methods include assessing nursing facility capabilities for technology adoption and use. Conducting many hours of observation of clinical staff to determine what communication strategies are being used and how technology does or does not support those strategies. Finally, we developed use cases based on our documented observations of different communication strategies and conducted semi-structured interviews with end users to validate the use cases and to discuss opportunities and challenges with implementing an HIE. These methods allowed us to effectively move our end users from a theoretical model of HIE use to a real implementation model that is currently being tested to improve $\mathrm{NH}$ resident outcomes.

\section{Clinical Relevance}

Health information exchange between health care providers has always been a crucial factor to deliver appropriate and timely care to patients. The push for technology adoption through meaningful use legislation has made the use of HIE even more important as healthcare organizations are striving to meet regulations. Descriptions of these new models of care incorporating HIE across the continuum can inform a wide array of stakeholders including administrators, clinicians, patients and family members about effective HIE implementation strategies to improve patient care communication. We have added several use cases to inform stakeholders how HIE can be implemented to improve patient care communication in the nursing home setting and beyond. Testing these use 
cases to determine their feasibility and impact, in our study of NH HIE adoption, is the next phase of our national demonstration project. Through a formative evaluation process we will be able to determine if the HIE implementation improved communication about resident care, what the strongest and weakest aspects of using HIE are in the NH setting, and how clinicians and other staff feel about using HIE as part of their work.

\section{Conflicts of Interest}

The authors declare that they have no conflicts of interest in this work.

\section{Protection of Human Subjects}

The study was performed in compliance with the World Medical Association Declaration of Helsinki on Ethical Principles for Medical Research Involving Human Subjects and was reviewed by University of Missouri’s Institutional Review Board.

\section{Acknowledgement}

This project is supported by grant number 1E1CMS331080 from the Centers for Medicare and Medicaid (CMS) Innovations Center. The content is solely the responsibility of the authors and does not necessarily represent the official views of CMS. 


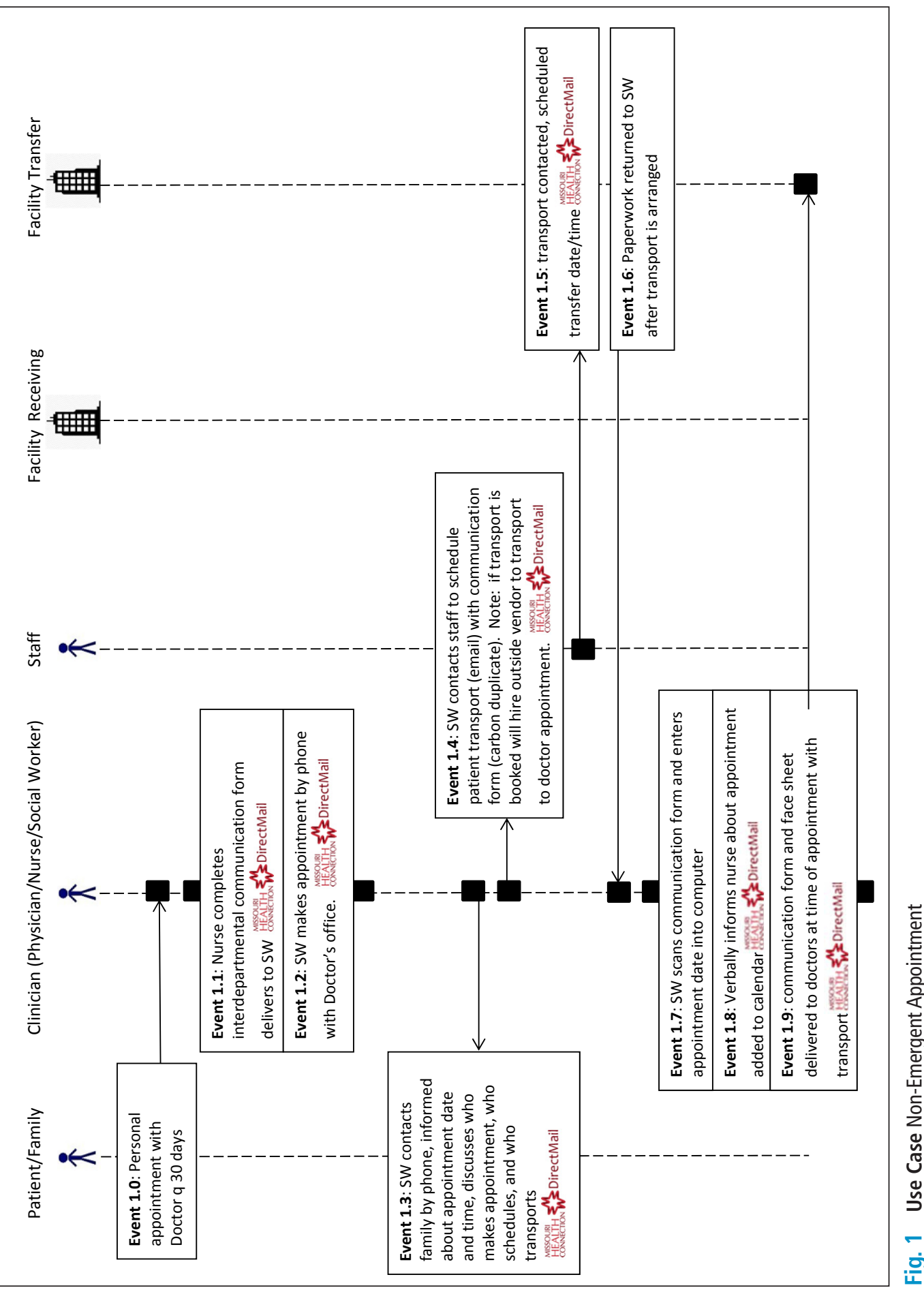

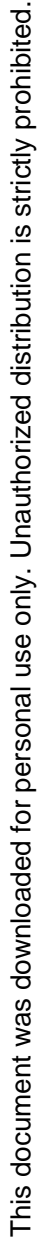




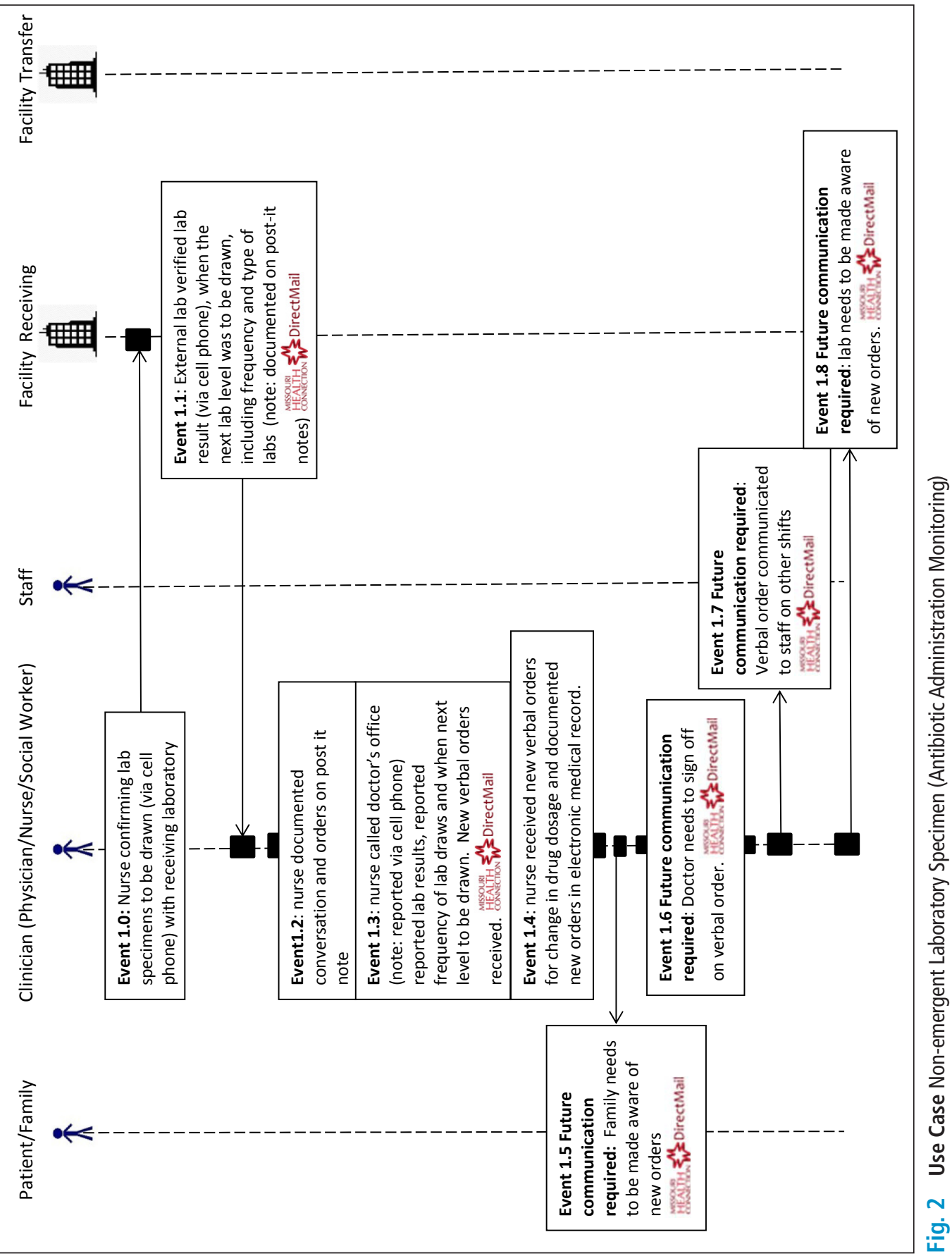




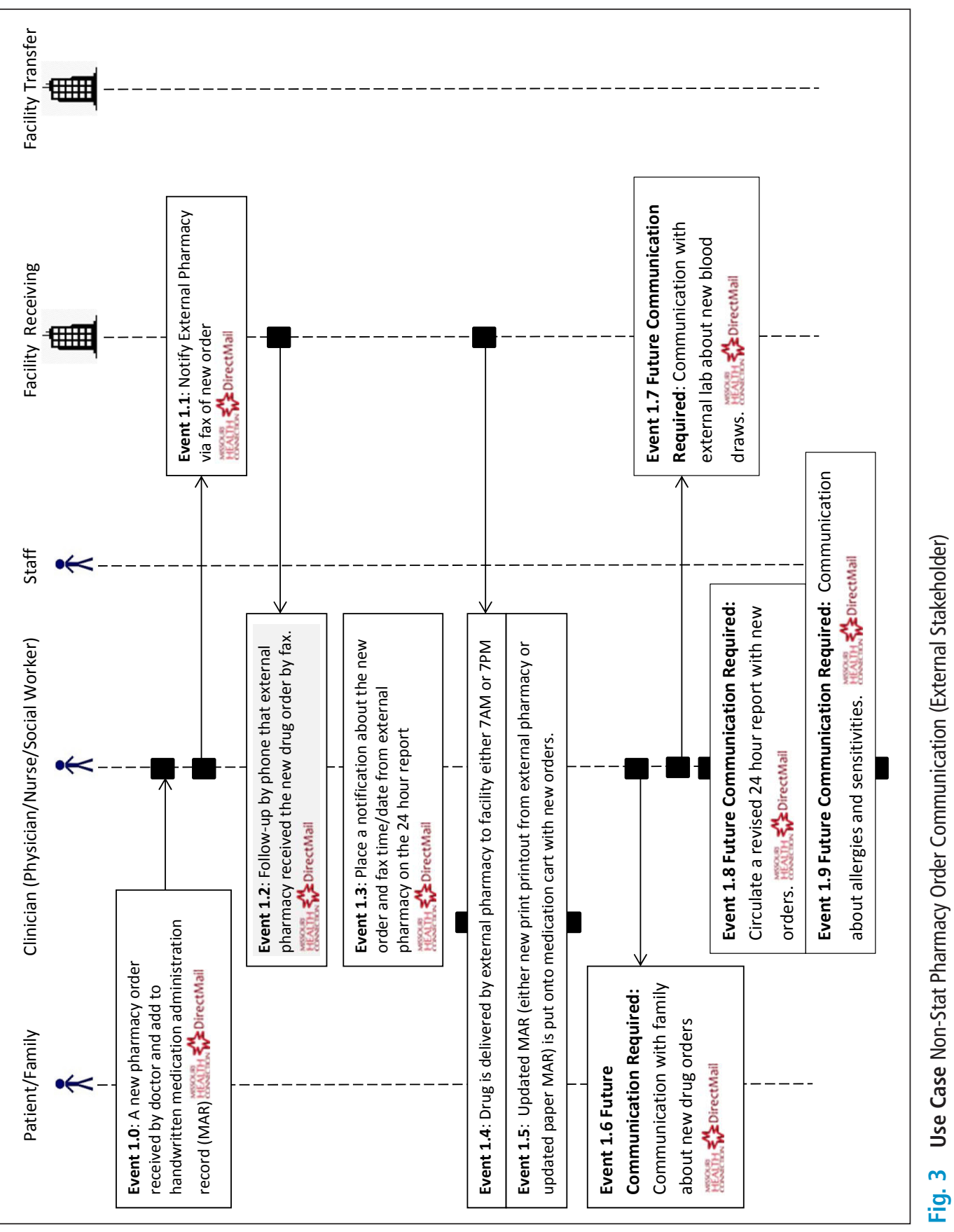




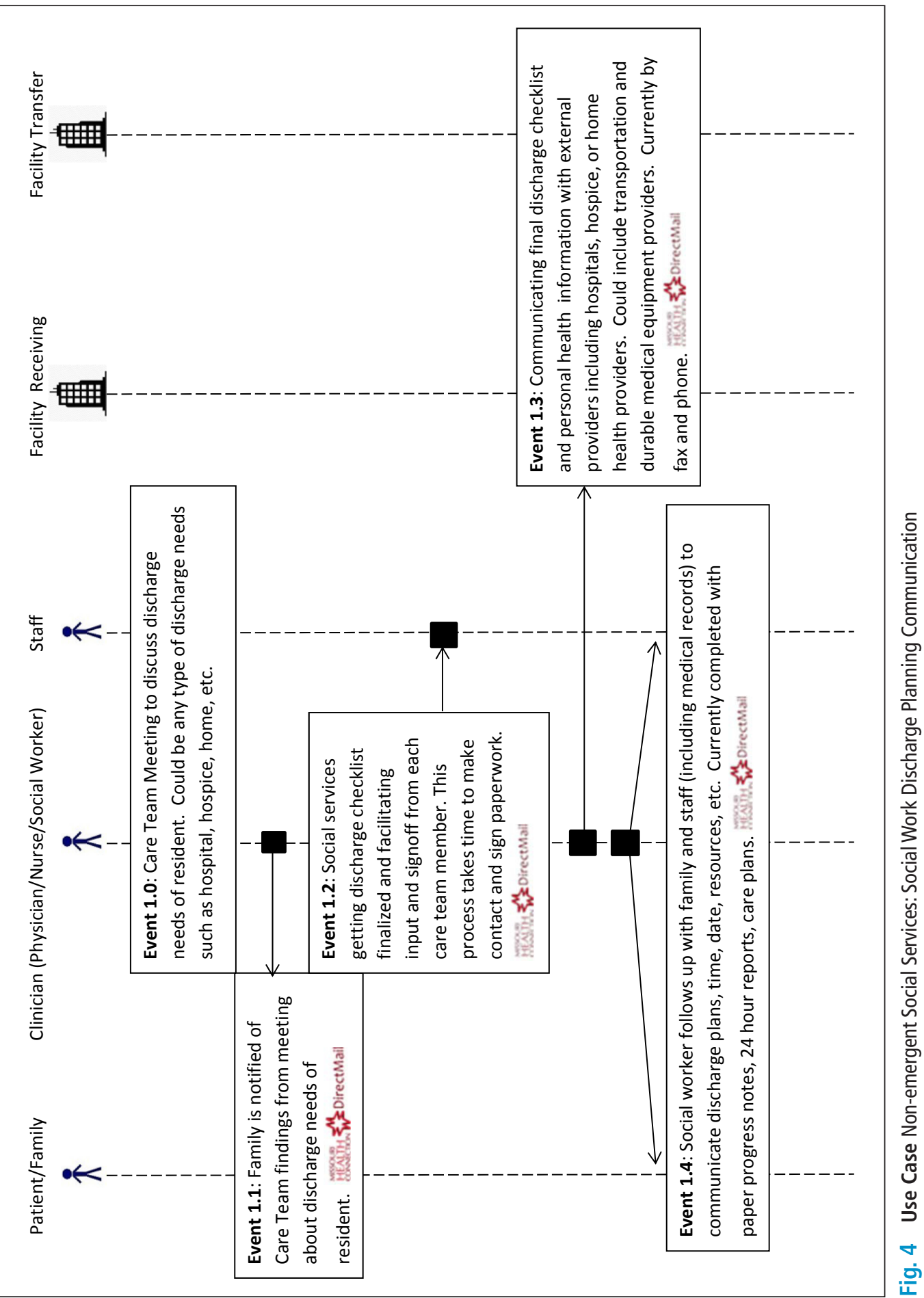




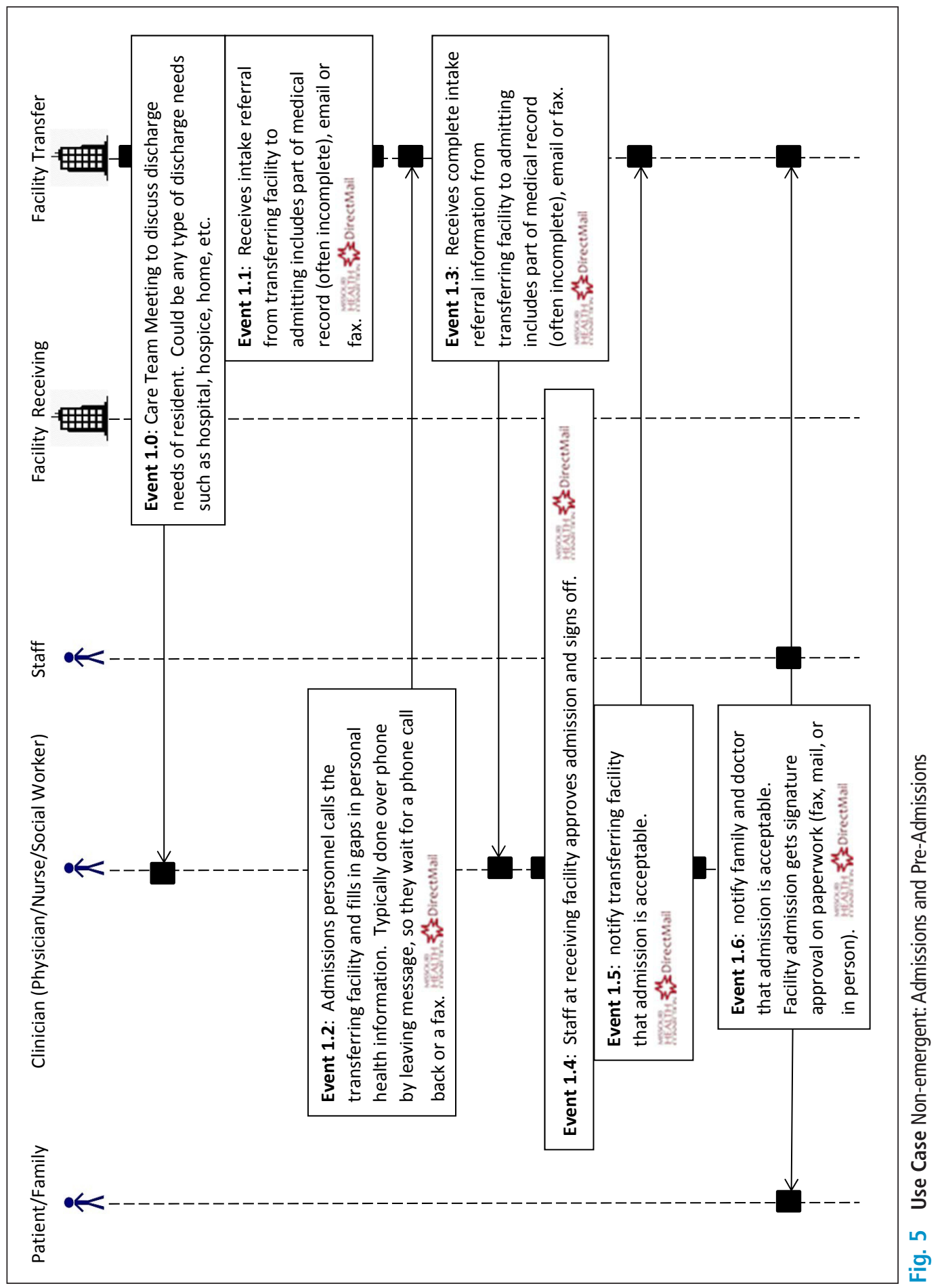

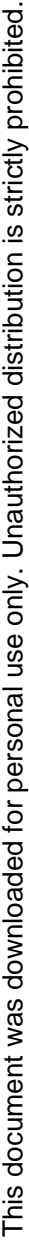




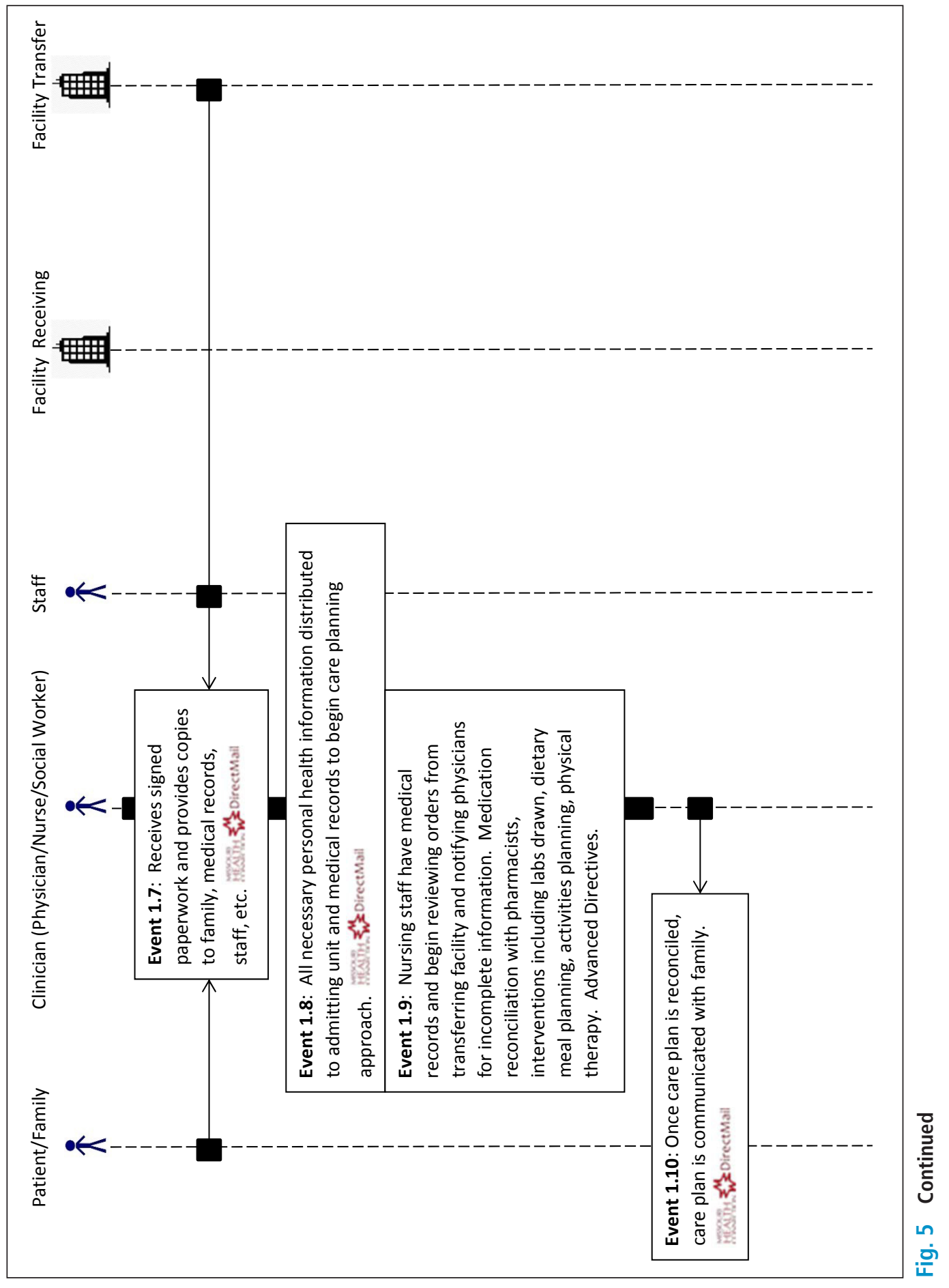




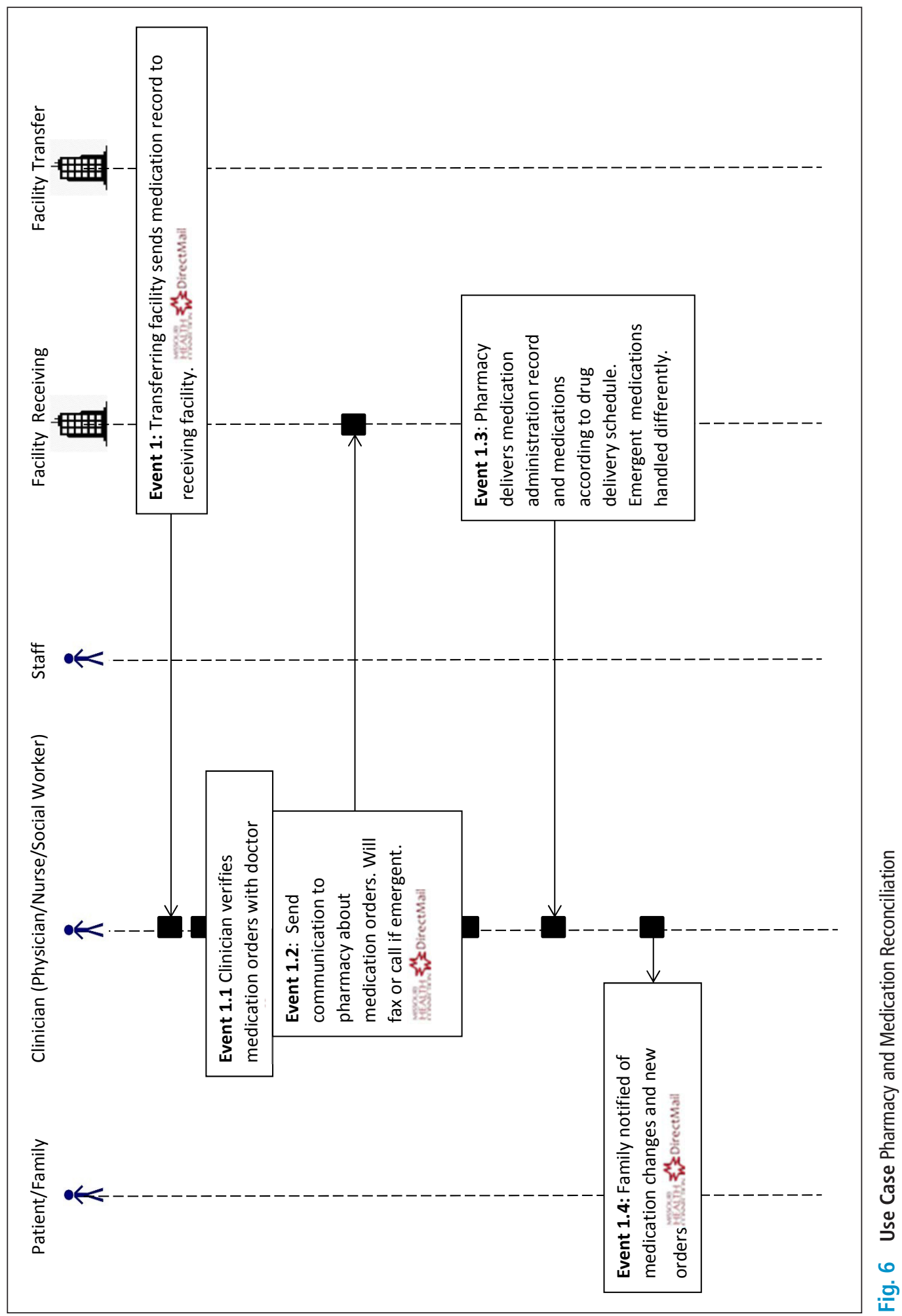


Table 1

\begin{tabular}{|c|c|c|c|c|c|c|c|}
\hline \multirow{2}{*}{$\begin{array}{l}\text { Please circle the answer that best indicates the ex- } \\
\text { tent to which } \\
\text { Medical or resident records are computerized in your nursing } \\
\text { home }\end{array}$} & \multicolumn{4}{|c|}{ Not at all } & \multicolumn{3}{|c|}{ Very much } \\
\hline & 1 & 2 & 3 & 4 & 5 & 6 & 7 \\
\hline $\begin{array}{l}\text { Your resident care systems are able to interface with external en- } \\
\text { tities' computerized systems (e.g., clinics, clinical laboratory, hos- } \\
\text { pitals, other nursing homes, etc.) }\end{array}$ & 1 & 2 & 3 & 4 & 5 & 6 & 7 \\
\hline \multicolumn{8}{|c|}{ Resident care systems are integrated with other computerized systems internal to your nursing home } \\
\hline - laboratory systems & 1 & 2 & 3 & 4 & 5 & 6 & 7 \\
\hline - PT/OT systems & 1 & 2 & 3 & 4 & 5 & 6 & 7 \\
\hline - dietary systems & 1 & 2 & 3 & 4 & 5 & 6 & 7 \\
\hline - pharmacy systems & 1 & 2 & 3 & 4 & 5 & 6 & 7 \\
\hline - human resources systems & 1 & 2 & 3 & 4 & 5 & 6 & 7 \\
\hline - finance systems & 1 & 2 & 3 & 4 & 5 & 6 & 7 \\
\hline \multicolumn{8}{|c|}{ Please check which of the following nursing processes or documents are computerized } \\
\hline $\begin{array}{l}\text { None are computerized } \\
\text { Staff scheduling } \\
\text { Vital signs recording (from monitoring equipment) } \\
\text { Medication administration } \\
\text { Staff workload management } \\
\text { Physician orders transcription } \\
\text { Care planning/RAPS } \\
\text { Historical record keeping } \\
\text { Resident acuity/condition recording } \\
\text { Quality assurance } \\
\text { Nursing flowsheet } \\
\text { Incident reporting } \\
\text { Real time or continuous MDS/RAI } \\
\text { Clinical reporting (e.g. treatments) } \\
\text { Other (s): }\end{array}$ & & & & & & & \\
\hline
\end{tabular}




\section{Reference List}

1. National Center for Health Statistics. Centers for Disease Control and Prevention FastStats Homepage. http://www cdc gov/nchs/fastats/nursingh htm 2011 February 18

2. Department of Health and Human Services. HealthIT.gov. http://www healthit gov/HIE 2012 March 14;Available from: URL: http://www.healthit.gov/HIE

3. Gaskin S, Georgiou A, Barton D, Westbrook J. Examining the role of information exchange in residential aged care work practices-A survey of residential aged care facilities. BMC Geriatrics 2012; 12(40): 1-11.

4. Georgiou A, Marks A, Braithwaite J, Westbrook J. Gaps, Disconnections, and Discontinuities-The Role of Information Exchange in the Delivery of Quality Long-Term Care. The Gerontologist 2013; 53(5): 770-779.

5. Rouse M. TechTarget: Personal Health Information. http://searchhealthit techtarget com/definition/personal-health-information 2015

6. Alexander GL, Steege L, Pasupathy K, Strecker EB. Case studies of IT sophistication in nursing homes: A mixed method approach to examine communication strategies about pressure ulcer prevention practices. International Journal of Industrial Engineering. In press, available on-line January 11, 2013.

7. Alexander GL. Nursing assistant communication strategies about pressure ulcers in nursing homes. Western Journal of Nursing Research 2015; 37.

8. DHHS. State HIE Bright Spots Implementation Brief. http://www publichealthok org/ files/4714/0310/6298/Oklahoma_LTPAC_Implementation_v_2_1 pdf 2013;Available from: URL: http://www.publichealthok.org/files/4714/0310/6298/Oklahoma_LTPAC_Implementation_v_2_1.pdf

9. Wang TBS. Adoption and utilization of electronic health record systems by long-term care facilities in Texas. Perspectives in Health Information Management 2012; 9(1g).

10. Abramson EL, McGinnis S, Moore J, Kaushal R, HITEC Investigators. A statewide assessment of electronic health record adoption and health information exchange among nursing homes. Health Services Research 2014; 49(1 Pt 2): 361-372.

11. Rantz MJ, Alexander GL, Galambos C, Vogelsmeier A, Popejoy L, Flesner M, Lueckenotte A, Crecelius C, Zwygart-Stauffacher M, Koopman RJ. Initiative to test a multidisciplinary model with advanced practice nurses to reduce avoidable hospitalizations among nursing facility residents. Journal of Nursing Care Quality 2014; 29(1): 1-8.

12. Kierkegaard P, Kaushal R, Vest JR. How could health information exchange better meet the needs of care practitioners? Applied Clinical Informatics 2014; 5(4): 861-877.

13. National Rural Health Association. HIE ToolKit. http://www ruralcenter org/rhitnd/hie-toolkit 2013 December 3

14. Centers for Medicaid and Medicare Services. Medicare Hospital Quality Chartbook. https://www cms gov/Medicare/Qulaity-Initiatives-Patient-Assessment-Instruments/HospitalQualityInits/Downloads/ HospitalChartBook2011 pdf 2011

15. Alexander GL, Wakefield DS. IT sophistication in nursing homes. Journal of the American Medical Directors Association 2009; 10(6): 398-407.

16. Alexander GL, Madsen R, Wakefield DS. A Regional Assessment of Information Technology Sophistication in Missouri Nursing Homes. Policy, Politics \& Nursing Practice 2010; 11(3): 214-225.

17. Wickens CD, Lee JD, Liu Y, Gordon-Becker SE. An Introduction to Human Factors Engineering. 2nd ed. Upper Saddle River, NJ: Pearson: Prentice Hall; 2004.

18. King BJ, Gilmore-Bykovskyi AL, Roiland RA, Polnaszek BE, Bowers BJ, Kind AJH. The consequences of poor communication during hospital to skilled nursing facility transitions: A qualitative study. Journal of the American Geriatrics Society 2013; 61(7).

19. Missouri Health Connection. MHC CareMail. http://www missourihealthconnection org/mhc-caremail-1 2015

20. Rudin RS, Motala A, Goldzweig CL, Shekelle PG. Usage and effect of health information exchange. Annals of Internal Medicine 2014; 161(11): 803-811.

21.Rantz MJ, Alexander GL, Galambos C, Flesner MK, Vogelsmeier A, Hicks L, Scott-Cawiezell J, ZwygartStauffacher M, Greenwald L. The use of bedside electronic medical record to improve quality of care in nursing facilities: A qualitative study. Computers Informatics Nursing 2011; 29(3): 149-156.

22. Alexander GL, Rantz MJ, Flesner M, Diekemper M, Siem C. Clinical information systems in nursing homes: An Evaluation of initial implementation strategies. Computers Informatics Nursing 2007; 25(4): 189-197.

23. Wright A, Soran C, Jenter CA, Volk LA, Bates DW, Simon SR. Physician attitudes toward health information exchange: Results of a statewide survey. Journal of the American Medical Informatics Association 2010; 17(1): 66-70. 
24. Rantz MJ, Hicks LL, Petroski GF, Madsen RW, Alexander GL, Galambos C, Conn V, Scott-Cawiezell J, Zwygart-Staffacher M. Cost, staffing and quality impact of bedside electronic medical record (EMR) in nursing homes. Journal of the American Medical Directors Association 2010; 11(7): 485-493.

25.Lyhne S, Georgiou A, Marks A, Tariq A, Westbrook J. Towards an understanding of the information dynamics of the handover process in aged care settings - a prerequisite for the safe and effective use of ICT. International Journal of Medical Informatics 2012; 81: 452-460.

26. Yu P, Li H, Gagnon M-P. Health IT acceptance factors in long-term care facilities: A cross sectional survey. International Journal of Medical Informatics 2009; 78: 219-229.

27. Mei YY, Marguard J, Jacelon C, DeFeo AL. Designing and evaluating an electronic patient falls reporting system: perspectives for the implementation of health information technology in long term residential care facilities. International Journal of Medical Informatics 2013; 82(11): e294-e306.

28. Kruse CS, Regier V, Rheinboldt KT. Barriers over time to full implementation of health information exchange in the United States. JMIR Medical informatics 2014; 2(2): e26.

29. Kruse CS, Mileski M, Alaytsev V, Carol E, Williams A. Adoption factors associated with electronic health record among long term care facilities: A systematic review. BMJ Open 2015; 5(1): e006615. 